\title{
A Review of Microsurgery Versus Endoscopy: Controversies for Treatment of Colloid Cysts
}

\author{
Alireza Razzaghi', Atefeh Yousefi², Sajjad Alizadeh ${ }^{2^{*}}$ \\ 'Safety Promotion and Injury Prevention Research Center, Shahid-Beheshti University of Medical Sciences, Tehran, Iran \\ ${ }^{2}$ Functional Neurosurgery Research Center, Shohada Tajrish Comprehensive Neurosurgical Center of Excellence, Shahid \\ Beheshti University of Medical Sciences, Tehran, Iran
}

\begin{abstract}
Background: There is controversy about the value of endoscopic methods compared to microsurgical methods in the treatment of challenging colloidal cysts. This study aimed to review the findings of literature which studied microsurgery or neuroendoscopy in the colloid cyst.

Methods: An advanced search in PubMed, Science Direct, and Google Scholar databases performed using keywords such as: "microsurgery," "endoscopy," "microsurgery versus endoscopy," and "colloid cysts."

Results: Reviewing the findings of related studies showed some differences in sections of surgical management, microsurgery, endoscopy, complete cyst resection, recurrence rate, length of stay, and complications between the two surgical methods in the treatment of colloid cyst.

Conclusion: Despite some disadvantages of endoscopy, it seems this method has more privileges than the other methods.

Keywords: Microsurgery; Endoscopy; Surgery; Colloid cysts.
\end{abstract}

*Correspondence to Sajjad Alizadeh, MD, Functional Neurosurgery Research Center, Shohada Tajrish Comprehensive Neurosurgical Center of Excellence, Shahid Beheshti University of Medical Sciences; Tel: +98(918)7467005; Email: sjd.alizadeh@gmail.com

Published online September 23, 2019

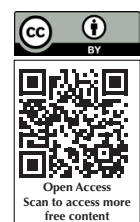

Citation: Razzaghi A, Yousefi A, Alizadeh S. A review of microsurgery versus endoscopy: controversies for treatment of colloid cysts. Int Clin Neurosci J. 2019;6(4):123-128. doi:10.15171/icnj.2019.23.

\section{Introduction}

Colloid cysts manifest symptoms due to ventricular obstruction or forniceal compression. Many surgeons and patients choose surgical resection. Transcortical or transcallosal microsurgery with craniotomy is the primary treatment for colloid cysts that prefers excellent access to the third ventricle and the foramen Monroe. As well as, it prefers two-handed microdissection of the cyst wall of essential structures like fornix, pleural choroid, and internal cerebral vessel. ${ }^{1-3}$ An association found between postoperative morbidities and craniotomy. ${ }^{4,5}$ However, recent clinicians prefer endoscopic surgery for the treatment of a colloid cyst. Studies report this technique as less invasive, leading to fewer complications and morbidities following surgery. ${ }^{6-16}$

On the other hand, a two-handed cyst wall microdissection is one of the essential structures discussed because this method relies on cyst wall aspiration, followed by coagulation of cyst wall residues remains. ${ }^{6}$ The remaining cyst wall increases the risk of recurrence in the future. ${ }^{8,10,12,16-18}$ Complete resection has frequently reported in microsurgery patients. While the shorter length of hospitalization and fewer complications mostly seen in endoscopic surgeries. ${ }^{3,4,16,19,20}$ Still, preferring the most appropriate surgical approach is controversial. This study aims to review the findings of literature which studied microsurgery or neuroendoscopy in the treatment of colloid cyst.

\section{Materials and Methods}

An advanced search in PubMed, Science Direct, and Google Scholar databases was performed using keywords such as "microsurgery," "endoscopy," "microsurgery versus endoscopy", and "colloid cysts." The abstract of every article was studied by researchers to determine the eligibility of this study, and then the full article was retrieved to extract the findings which were summed up in the following sections: surgical management, microsurgery, endoscopy, complete cyst resection, recurrence rate, length of stay and complications.

\section{Endoscopic Versus Microsurgical Surgery \\ Surgical Management}

Extensive research on the literature revealed that microsurgical cutting and endoscopic resection are the most popular ways for the treatment of colloid cysts. ${ }^{6,20,21}$ Preferring each of these techniques over the other depends on the surgeon's skill. Using microsurgical 
techniques decreases the morbidity and mortality rates significantly. ${ }^{22,-25}$ Studies have reported the widespread use both of transcortical and transcallosal techniques for third ventricle colloid cysts treatment. ${ }^{6,13,21,26-32}$ Though many clinicians consider transcranial microsurgery as the touchstone for removal of colloid cysts, ${ }^{6,8}$ still, there is no consensus on the best optimal treatment for patients with colloid cysts and normal. Endoscopic treatment of colloid cysts in the presence of ventriculomegaly has established

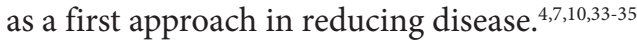

\section{Microsurgery}

Colloid cysts are treated by either microsurgical removal using either transcallosal or transfrontal techniques. ${ }^{7,36}$ Open microsurgery needs a more significant access corridor, but total resection is the advantage. . $^{13,32,34}$ Compared to endoscopic approaches, these techniques suggest a better perspective of the cyst so that it facilitates full cut. Many studies have reported the feasibility of better visualization of the cyst by an endoscope with angled optics. There is little difference in the benefits of using the instrument during microscopy compared to endoscopic surger. ${ }^{6}$ previously, microsurgical resection was the best preference for curing patients with colloid cysts. The transcortical-transventricular technique sometimes leads to high morbidity and mortality. ${ }^{32}$ The transcallosal method rescues the cortex but can damage the bridging veins, sinuses, and pericallosal arteries and may also injure the fornix. Though highly invasive, transcortical, or transcallosal microscopy has advantages over endoscopy in critical situations. The former has the potential complications related to anterior callosotomy and transcortical techniques with further damage to the cortex and increased risk of seizures. Although endoscopic surgery is less offensive, bleeding is difficult to control in this procedure. Therefore, an irrigation pump with a Ringer's solution is necessary to irrigate periodically for subsequent washing and discharge through the endoscope outlet work channel. Endoscopic surgery is superior only after reaching the learning curve, because the potential complications and the rate of failure may be high in the beginner surgeon. ${ }^{14}$ Microscopic surgery is faster than the original resection but is associated with longer operation time and more significant memory and cognitive dysfunction. ${ }^{2}$

\section{Endoscopy}

Endoscopic removal of colloid cysts is adequately safe. ${ }^{7,10,12,18,30,37}$ It is a relatively novel method providing remarkable imagination and "a minimal invasive passage via the normal expanded ventricular system." This approach has the benefit of controlled resection, which prevents any damage to the anterior third ventricle. Complete resection using endoscopy, though difficult, is achievable. Near-total excision is possible with one port or by conventional endoscopic procedures. ${ }^{6,10,12,35}$ This new way allows all segments to recover and finally leads to full cyst removal. ${ }^{38}$ Endoscope could be placed in a way to reinforcement microsurgical availability, minimize the size of the transcortical passages, and decrease brain contraction when removing intraventricular damaged tissues. There are some studies on good results after endoscopic resection of colloid cysts in the third ventricle. ${ }^{39}$ Scientists have rejected using intraventricular endoscopy in the absence of hydrocephalus and suggested using frameless stereotaxis to help surgeons to create a perfect rush corner. The latter subject includes a sufficient work zone in the ventricle. The ventriculomegaly existence comfort the ventricle function. Still, its loss does not prevent using endoscopy of the lesions. Some studies showed favorable results of endoscopy with small ventricles. ${ }^{17,39}$ In this procedure, a seal created about the endoscope, which prevents the emergence of cerebrospinal fluid (CF) and ventricular decline, so CF flows slowly from one of the endoscope ports. This prevents inducing hypertension within the skull. ${ }^{10}$ The endoscope has better lighting than an endoscope to observe the third ventricle and the fornix underside.

Furthermore, hospitalization is shorter than the other much invasive surgery method. ${ }^{6}$ According to prior investigations, colloid cysts considered as the central cause of quick and vast neurological damage and sudden death. However, endoscopy reduces the duration of surgery, and patients will face fewer complications due to smaller cortical incision and possible seizures. ${ }^{8}$ Clinician report that a single portal endoscopic procedure is suitable for tumor resection in most sufferers, but the surgical equipment transported to the same level of vision as the endoscope meaning that their action was as well as the video image motion so the surgeons cannot have a perfect tridimensional view of the operational field. However, research ignored this as a primary issue "since exact tridimensional signs achieved since the endoscope endlessly resituated in the ventricle." By inserting a latter contiguous portal, endoscopic tools can be "handled the endoscope independently." This is similar to the strategy utilized in other endoscopic surgical proficiencies in which two or more availability portals were usually utilized..$^{33}$ There are advantages in endoscopic approaches because the patient is positioned neutrally supine (thorax lifts with 15 points).

Moreover, the cutting and burr hallow track the landmarks on a standard right anterior ventriculostomy and a direct section, and one burr hollow enables fast achievement to the cyst. The endoscope is inserted via a presenter, some bigger than a ventricular catheter. Therefore there is very little disorder in the cortex. More importantly, local anesthesia is possible in this method. The other benefits of endoscopic operation include the changeable corner of embodiment and brightness contrast to operative and minimally invasive microscope. Thus, endoscopy reduces operation time, 
and hospitalization, and post-operative complaints, and no remarkable memory and cognitive deficits often manifest; however, a superior measure of imperfect removal is observed. ${ }^{4,17,19,39,40}$ Later, parallel endoscopy developed as a more beneficial method to improve visualization. ${ }^{41}$ Stereotactically Guided Endoscopic Port Surgery is said to be a mixture of four novel technologies to enhance the quality of the minimally invasive neurosurgery, so it comprises endoscopy, stereotactic guidance, microsurgery, and intraoperative imaging. However, the surgery room furnished with a 64-slice CT scanner, new endoscopic tools with observation monitors, stereotactic projecting software, and a proprietary surgery room group. Endoscopy has mainly associated with low morbidity in small series. ${ }^{7,8,10,12,18,19,33,34,40}$ Many studied have proved this method for total colloid cyst excision. . $2,33-35,40,41^{-1}$ Microsurgical resection preferred in some rare situations under specific circumstances.

\section{Complete Cyst Resection}

The transcranial microsurgery, either transcallosal or transcortical route, is the most popular used procedures for removing colloid cysts. ${ }^{4}$ A critique in the endoscopic technique is its inability to remove cysts thoroughly compared to open ways. The removing amount differs vastly. ${ }^{36}$ In a category, the cysts were removed entirely in $80 \%$ of the patients who had been cured endoscopically. ${ }^{33}$ Although the following cycle in these categories was long, a longer follow cycle suggested because recurrence and complications due to incomplete resection are possible. ${ }^{6}$ So far, surgeons have suggested the following treatments for the patients with colloid cysts in the third ventricle: microsurgical removing, stereotactic inhalation, endoscopic removing, and using ventriculoperitoneal shunt. Some believe that as time goes on, experience helps surgeons to go on with endoscopic techniques with total resections, though epilepsy, memory loss, hemiparesis, cerebellar hemorrhage, Terson's syndrome as well as some other complications have been recorded. ${ }^{8,19,39}$ Also, hypothalamic hamartoma removing from the third ventricle has related to great results in patients with normal ventricles. According to several experiences, all third ventricular colloid cysts should not be operated endoscopically. ${ }^{6}$ Resection considered complete if intraoperative assessment and post-surgery MRI showed the total elimination of cyst. ${ }^{6}$ Lately, Decq et al announced their experiment of endoscope colloid cysts and aspirating its contents. Sometimes when a small tissue part is inseparable of neurotic or vascular structure, this must be clotted and remained intact. In other fields, an endoscopic bipolar clotting system incorporated that it has obvious benefits in unipolar cautery or laser clotting, which lets somewhat accurate microendoscopic gradual removing of tumors and clotting with electrical running extension into the neighbor structure. ${ }^{42}$ Removing of endoscopic colloid cyst might be difficult and not possible at specified conditions. Initial, the existence of a tiny ventricle is a unique, strict condition and strictly troublesome. ${ }^{42}$ Surgical removing mostly selected on large symptomatic colloid cysts. ${ }^{1-3}$ Transcortical and transcallosal microsurgery in craniotomy have ever been the main pillars for surgeons to treat colloid cyst. ${ }^{4,5,7}$ Microsurgical fields have generally described superior levels of full removing. ${ }^{2,3,9}$ Before any treatment, illness related to origin surgical removing and relapse possibility should be considered well enough. ${ }^{8,10}$ Some investigators put that entire cyst wall removing is necessary. Otherwise, the cyst would have a relapse. ${ }^{13}$ Some rely on mere postsurgery imaging, not intraoperative inspection. Still, others prefer total mass aspirations and debris cyst wall clotting in the role of gross total resection. ${ }^{8,12,14}$ At endoscopy, it is an uncommon incidence that total cyst wall debris would full take from somewhere it has joined to choroid plexus and internal cerebral vein in the third ventricle roof. Cyst wall clotting should not place below the class of full removing, because wall debris will ever stay in the role of a possible origin for relapse. Beside, microsurgery lets the surgeon carry out a proper autopsy of the cyst wall far from an acute concrete structure using two-handed microsurgical methods to attain full cutting, so it has a superior level of gross total resection, less level of relapse and re-surgery level. ${ }^{3}$ Failure to obtain full removing can happen for various causes include anatomical remarks and the surgeon's practical experiment and proficiency. ${ }^{5}$ Some authors still doubt about the complete removal of the cyst capsule. ${ }^{2,13}$ The nature of endoscopy inhibits the complete removal of it compared to microsurgery. ${ }^{2}$ In an endoscopic operated group, incomplete cyst resection was high $;^{16}$ however, subtotal resection and cyst recurrence have been reported even in microsurgical series. ${ }^{39}$ Modern technology in endoscopes design comprise more important ports for tools, and broad-angle lenses must aid reduction in the level of remaining cysts. ${ }^{20}$

\section{Recurrence Rate}

Residual cyst occurring will enhance the risk of potential recurrence. ${ }^{14,18,19}$ Although an endoscopic removing role in colloid cysts therapy supported for secure as well as efficient quality, the other studies showed complete resection in some cases may involve a change to the apparent method. ${ }^{26,27}$ Although adequate assessment of the clinical importance of cyst residues after endoscopic removal has not performed, it suggested that endoscopic resection is associated with a higher probability of recurrence. This paper reflects concern over the importance of the higher rate of cyst residues after endoscopic resection. For instance, Dr. Carl Heilman has expressed that "partial removal of the colloid cyst wall will not lead to enduring treatment. ${ }^{18}$ Although utilizing endoscope appeared encouraging, the recurrence rate has been a matter of concern. ${ }^{17}$ Up to now, relapse has described in cases in which a well-known remnant cyst 
observed in addition to the cyst wall, which removed incompletely, and residues clotted. ${ }^{1,2,20}$

Length of Stay

Naturally, hospitalization duration was less to some extent in the endoscopy team than in transcallosal craniotomy one. Though it has not particularly examined, this distinction can converse to more economical therapy. ${ }^{17}$ Microsurgery is mostly related to hospitalization length and illness higher levels. ${ }^{9,18,20,26,29}$ These patients require close enduring following should a remnant cyst stays, since conducting the most strictly prosperous endoscopic methods. Several studies declared that fewer patients' hospitalization may result in more affordable. ${ }^{17}$

\section{Operative or Neurologic Complications}

There is no consensus among surgery team members regarding the incidence of complications. Despite these difficulties, the vast number of patients recuperated enough to be dismissed to home. ${ }^{17}$ Some investigators have also reported venous infarction after thrombosis from cortical veins. ${ }^{21,27}$ However, which kind of difficulty would not happen in a transcortical way, some surgeons tend to choose the transcallosal method, which refrains from harm to cortical tissue. Whenever a picture guide used, nevertheless, a right frontal transcortical method beseems to be related to a seldom difficulty profile and is a rational option. In other fields, using transcallosal or transcortical methods have related to brain disorder. ${ }^{22,29}$ Despite being a minimum invasion, endoscopy is yet related to complications. Intense complications, such as hemiparesis and brain defects, can happen. ${ }^{19,26,42}$ Open microsurgical cutting out commonly brought up standard golden therapy. Operating methods are risky. ${ }^{32}$ The transcallosal method refrains from the cortical cutting risk. However, it is related to complications that comprise cortical venous infarction, forniceal destruction, damage to the deep venous system, subdural hematoma, disjunction syndromes, ventriculitis, and meningitis. ${ }^{7,30}$ Minimum invasion methods tried to find minimizing surgery effect and involve the cyst contents aspiration. ${ }^{33}$ The primary investigator also announced a notably less risk of complications in endoscopic than the microsurgical removing of colloid cysts with an equal elimination level. ${ }^{31,37}$ A relationship has found between cutting out of the endoscopic colloid cyst and a downhill acquisition curve. The complication level of endoscopic neurosurgery is little, but life-minatory complications, though uncommon, can happen. The complication level could reduce significantly with surgical experiments and instruction. ${ }^{32}$ Hydrocephalus, loss of vision, endocrine impairment, and behavior, and cognitive impairments are popular representations and potential surgical side-effects. Common and stereotactic microsurgical methods involve the transfrontal, transcortical, and transcallosal paths to the ventricular. ${ }^{24,30,35}$ The availability of transcortical intraventricular mostly needs to considerable brain contraction, despite using no frame picture guide to predict a path. Side-effects of the above methods involve seizures, focal neurological impairments, and cognitive defects sometimes associated with venous infarction. ${ }^{6,36}$ Forniceal harm is a possibly destructive side-effect related to both microsurgical and endoscopic colloid cyst removing. ${ }^{38}$ On prior open and endoscopic systems, memory loss has ranged from $20 \%$ to $30 \% .^{10,11,17,19,26,32,42}$ Although tumors can be destroyed thoroughly by microsurgery in most patients, side-effects have further commonly described than fewer invasion methods. These side-effects involve the risks that protect frontal lobe contraction or cutting out and potential damage to deep venous and neural constructions, as the internal cerebral veins and fornices. The stereotactic hole is encouraging since this is a minimum invasion, but limit since colloid material may be excessively viscous or solid, to make aspiration unfeasible. Also, neural or vascular constructions like choroid plexus can engulf the lesion, or the big vena in the venous corner can cover it that may not comprehend in stereotactic surgery. Commonly, cyst and neighboring texture should be anatomized or clotted for sufficiently subjecting tumors to become feasible, removing safely. The weak hole of the colloid cyst would endanger the constructions. ${ }^{2}$ Endoscopic systems have described less hospitalization, and reduced side-effect levels have been achieved. ${ }^{18,41}$ The total illness level was less in the endoscopic team $(10.5 \%)$ compared with microsurgery $(16.3 \%)$, according to the results of some studies. It was interesting that in the microsurgery team, the transcortical method had a higher total illness level $(24.5 \%)$ toward that in the transcallosal method (14.4\%). ${ }^{18}$ Endoscopy still involves complications, despite least invasiveness. Indeed, severe complications like hemiparesis and memory deficits can take place. ${ }^{2,42}$ In other series, memory impairment also resulted from using transcortical or transcallosal approaches., ${ }^{90,31}$ The major shortcomings in the endoscopic method encountered the acquisition curve for accomplishing skill, having difficulty on the use of hands concurrently to perform, and repetitive lens unclear. An anatomical restriction of radical cutting is cyst linking to the membrane interpositum in endoscopic technique; identification and visualization of the cyst are very hard via a precoronal node cavity much as velum interpositum be located a few centimeter frontals the coronal stitch. By providing that tough and rigid content, the endoscopic approach could be harder than an open microsurgical approach; currently use of proficiency instruments as an ultrasonic aspirator could solve the problem. ${ }^{12}$ Furthermore, endoscopy is mainly criticizing as follows. Firstly, damage to fornix around the foramen of Monro in this approach may result from the endoscopic sheath manipulation rather than in a microsurgical one. Secondly, thermal injuries may induce by extreme electrocoagulation. ${ }^{1}$ The patients 
treated with microsurgical techniques showed higher complications than those who underwent endoscopic approaches. ${ }^{14,42}$ Recently, a meager rate of mortality and complications has reported by the endoscopic approach in some articles. ${ }^{14,22,26}$ The major drawback of endoscopic treatment, conducted through a classic precoronal route, is the complexity in controlling the cyst adhesion to the tela choroidea and in deleting the cyst. ${ }^{14,23}$ It has found that the cyst attachment to the third ventricle roof limits the endoscopy. ${ }^{23}$ The major shortcomings intrinsic on the endoscopic approach are encountered acquisition curve accomplish skill, having difficulty use of hands concurrently for conducting, and repeated lens unclear. ${ }^{28}$

\section{Conclusion}

The new minimally invasive method, Endoscopic approach, makes direct tumor visualization possible irrespective of cyst size. It leaves little morbidity and presents favorable outcomes. Along with other technologies, endoscopic techniques have developed remarkable and now accepted as an alternative for microsurgical methods. Still, early post-operative residues and recurrences have created doubts upon its effectiveness times after the operation, so more research proposed to perform considering the recent experiences on both procedures across the world.

\section{Conflict of Interest Disclosures}

The authors declare that they have no conflict of interests.

\section{Ethical Statement}

Not applicable.

\section{References}

1. Pollock BE, Huston J 3rd. Natural history of asymptomatic colloid cysts of the third ventricle. J Neurosurg 1999;91(3):364-9. doi: 10.3171/jns.1999.91.3.0364.

2. Pollock BE, Schreiner SA, Huston J 3rd. A theory on the natural history of colloid cysts of the third ventricle. Neurosurgery. 2000;46(5):1077-81. doi: 10.1097/00006123-20000500000010

3. Wilson DA, Fusco DJ, Wait SD, Nakaji P. Endoscopic resection of colloid cysts: use of a dual-instrument technique and an anterolateral approach. World Neurosurg. 2013;80(5):57683. doi: 10.1016/j.wneu.2012.07.014.

4. Sampath R, Vannemreddy P, Nanda A. Microsurgical excision of colloid cyst with favorable cognitive outcomes and short operative time and hospital stay: operative techniques and analyses of outcomes with review of previous studies. Neurosurgery. 2010;66(2):368-74. doi: 10.1227/01. neu.0000363858.17782.82.

5. Bergsneider M. Complete microsurgical resection of colloid cysts with a dual-port endoscopic technique. Neurosurgery. 2007;60(2 Suppl 1):ONS33-42. doi: 10.1227/01. neu.0000249227.82365.36.

6. Wilkins RH, Rengachary SS. Neurosurgery. New York: McGraw Hill; 1985. p. 1419-34.

7. Hernesniemi J, Leivo S. Management outcome in third ventricular colloid cysts in a defined population: a series of 40 patients treated mainly by transcallosal microsurgery. Surg Neurol. 1996;45(1):2-14. doi: 10.1016/00903019(95)00379-7.
8. Grondin RT, Hader W, MacRae ME, Hamilton MG. Endoscopic versus microsurgical resection of third ventricle colloid cysts. Can J Neurol Sci. 2007;34(2):197-207. doi: 10.1017/s0317167100006041.

9. Kehler U, Brunori A, Gliemroth J, Nowak G, Delitala A, Chiappetta F, et al. Twenty colloid cysts--comparison of endoscopic and microsurgical management. Minim Invasive Neurosurg. 2001;44(3):121-7. doi: 10.1055/s-2001-18122.

10. Desai KI, Nadkarni TD, Muzumdar DP, Goel AH. Surgical management of colloid cyst of the third ventricle--a study of 105 cases. Surg Neurol. 2002;57(5):295-302. doi: 10.1016/ s0090-3019(02)00701-2.

11. Rodziewicz GS, Smith MV, Hodge CJ Jr. Endoscopic colloid cyst surgery. Neurosurgery. 2000;46(3):655-60. doi: 10.1097/00006123-200003000-00025.

12. Levine NB, Miller MN, Crone KR. Endoscopic resection of colloid cysts: indications, technique, and results during a 13year period. Minim Invasive Neurosurg. 2007;50(6):313-7. doi: 10.1055/s-2007-993215.

13. Hoffman CE, Savage NJ, Souweidane MM. The significance of cyst remnants after endoscopic colloid cyst resection: a retrospective clinical case series. Neurosurgery. 2013;73(2):233-7. doi: 10.1227/01. neu.0000430300.10338.71.

14. Boogaarts HD, Decq P, Grotenhuis JA, Le Guerinel C, Nseir $R$, Jarraya $B$, et al. Long-term results of the neuroendoscopic management of colloid cysts of the third ventricle: a series of 90 cases. Neurosurgery. 2011;68(1):179-87. doi: 10.1227/ NEU.0b013e3181ffae71.

15. Sheikh AB, Mendelson ZS, Liu JK. Endoscopic versus microsurgical resection of colloid cysts: a systematic review and meta-analysis of 1,278 patients. World Neurosurg. 2014;82(6):1187-97. doi: 10.1016/j.wneu.2014.06.024.

16. Abdou MS, Cohen AR. Endoscopic treatment of colloid cysts of the third ventricle. Technical note and review of the literature. J Neurosurg. 1998;89(6):1062-8. doi: 10.3171/ jns.1998.89.6.1062.

17. Horn EM, Feiz-Erfan I, Bristol RE, Lekovic GP, Goslar PW, Smith KA, et al. Treatment options for third ventricular colloid cysts: comparison of open microsurgical versus endoscopic resection. Neurosurgery. 2007;60(4):613-8. doi: 10.1227/01. neu.0000255409.61398.ea.

18. Greenlee JD, Teo C, Ghahreman A, Kwok B. Purely endoscopic resection of colloid cysts. Neurosurgery. 2008;62(3 Suppl 1):51-5. doi: 10.1227/01.neu.0000317373.00018.6f.

19. Hellwig D, Bauer BL, Schulte M, Gatscher S, Riegel T, Bertalanffy $H$. Neuroendoscopic treatment for colloid cysts of the third ventricle: the experience of a decade. Neurosurgery. 2003;52(3):525-33. doi: 10.1227/01. neu.0000047671.27057.55.

20. Hernesniemi J, Romani R, Dashti R, Albayrak BS Savolainen S, Ramsey C 3rd, et al. Microsurgical treatment of third ventricular colloid cysts by interhemispheric far lateral transcallosal approach--experience of 134 patients. Surg Neurol. 2008;69(5):447-53. doi: 10.1016/j. surneu.2007.11.005.

21. Garrido E, Fahs GR. Cerebral venous and sagittal sinus thrombosis after transcallosal removal of a colloid cyst of the third ventricle: case report. Neurosurgery. 1990;26(3):540-2. doi: 10.1097/00006123-199003000-00028.

22. Aggleton JP, McMackin D, Carpenter K, Hornak J, Kapur N, Halpin S, et al. Differential cognitive effects of colloid cysts in the third ventricle that spare or compromise the fornix. Brain. 2000;123(Pt 4):800-15. doi: 10.1093/brain/123.4.800.

23. Hodges JR, Carpenter K. Anterograde amnesia with fornix damage following removal of IIIrd ventricle colloid cyst. J Neurol Neurosurg Psychiatry. 1991;54(7):633-8. doi: 10.1136/jnnp.54.7.633.

24. Abernathey CD, Davis DH, Kelly PJ. Treatment of colloid 
cysts of the third ventricle by stereotaxic microsurgical laser craniotomy. J Neurosurg. 1989;70(4):525-9. doi: 10.3171/ jns.1989.70.4.0525.

25. Cabbell KL, Ross DA. Stereotactic microsurgical craniotomy for the treatment of third ventricular colloid cysts. Neurosurgery. 1996;38(2):301-7. doi: 10.1097/00006123199602000-00013.

26. Lewis Al, Crone KR, Taha J, van Loveren HR, Yeh HS, Tew JM Jr. Surgical resection of third ventricle colloid cysts. Preliminary results comparing transcallosal microsurgery with endoscopy. J Neurosurg. 1994;81(2):174-8. doi: 10.3171/ jns.1994.81.2.0174.

27. Mathiesen T, Grane P, Lindquist C, von Holst H. High recurrence rate following aspiration of colloid cysts in the third ventricle. J Neurosurg. 1993;78(5):748-52. doi: 10.3171/ jns.1993.78.5.0748.

28. Jeeves MA, Simpson DA, Geffen G. Functional consequences of the transcallosal removal of intraventricular tumours. J Neurol Neurosurg Psychiatry. 1979;42(2):134-42. doi: 10.1136/jnnp.42.2.134.

29. McMackin D, Cockburn J, Anslow P, Gaffan D. Correlation of fornix damage with memory impairment in six cases of colloid cyst removal. Acta Neurochir (Wien). 1995;135(12):12-8. doi: 10.1007/bf02307408.

30. Apuzzo ML, Chikovani OK, Gott PS, Teng EL, Zee CS, Giannotta SL, et al. Transcallosal, interfornicial approaches for lesions affecting the third ventricle: surgical considerations and consequences. Neurosurgery. 1982;10(5):547-54. doi: 10.1227/00006123-198205000-00001.

31. Stachura K, Libionka W, Moskala M, Krupa M, Polak J. Colloid cysts of the third ventricle. Endoscopic and open microsurgical management. Neurol Neurochir Pol. 2009;43(3):251-7.

32. Schroeder HW, Oertel J, Gaab MR. Incidence of complications in neuroendoscopic surgery. Childs Nerv Syst. 2004;20(1112):878-83. doi: 10.1007/s00381-004-0946-y.

33. Bosch DA, Rahn T, Backlund EO. Treatment of colloid cysts of the third ventricle by stereotactic aspiration. Surg Neurol. 1978;9(1):15-8.

34. Morita A, Kelly PJ. Resection of intraventricular tumors via a computer-assisted volumetric stereotactic approach. Neurosurgery. 1993;32(6):920-6. doi: 10.1227/00006123199306000-00006.

35. Yamamoto I, Rhoton AL Jr, Peace DA. Microsurgery of the third ventricle: Part I. Microsurgical anatomy. Neurosurgery. 1981;8(3):334-56. doi: 10.1227/00006123-19810300000006.

36. Rhoton AL Jr, Yamamoto I, Peace DA. Microsurgery of the third ventricle: Part 2. Operative approaches. Neurosurgery. 1981;8(3):357-73. doi: 10.1227/00006123-19810300000007.

37. Vialogo JG. [Endoscopic transepto-interforniceal approach to colloid cysts: case report]. Arq Neuropsiquiatr. 2000;58(3B):939-46. doi: 10.1590/s0004$282 \times 2000000500026$.

38. Wait SD, Gazzeri R, Wilson DA, Abla AA, Nakaji P, Teo C. Endoscopic colloid cyst resection in the absence of ventriculomegaly. Neurosurgery. 2013;73(1 Suppl Operative):ons39-46.doi:10.1227/NEU.0b013e3182870980.

39. Delitala A, Brunori A, Russo N. Supraorbital endoscopic approach to colloid cysts. Neurosurgery. 2011;69(2 Suppl Operative):ons176-82. doi: 10.1227/ NEU.Ob013e318219563c.

40. Teo C. Complete endoscopic removal of colloid cysts: issues of safety and efficacy. Neurosurg Focus. 1999;6(4):e9.

41. Gaab MR, Schroeder HW. Neuroendoscopic approach to intraventricular lesions. J Neurosurg. 1998;88(3):496-505. doi: 10.3171/jns.1998.88.3.0496.

42. King WA, Ullman JS, Frazee JG, Post KD, Bergsneider M. Endoscopic resection of colloid cysts: surgical considerations using the rigid endoscope. Neurosurgery. 1999;44(5):1103-9. doi: 10.1097/00006123-199905000-00090. 